\title{
Some results on caps and codes related to orthogonal Grassmannians - a preview
}

\author{
I. Cardinali ${ }^{1}$ \\ Dipartimento di Ingegneria dell'Informazione \\ Università di Siena \\ Siena, Italy \\ L. Giuzzi ${ }^{2}$ \\ Dipartimento di Matematica \\ Università di Brescia \\ Brescia, Italy
}

\begin{abstract}
In this note we offer a short summary of some recent results, to be contained in a forthcoming paper [4], on projective caps and linear error correcting codes arising from the Grassmann embedding $\varepsilon_{k}^{g r}$ of an orthogonal Grassmannian $\Delta_{k}$. More precisely, we consider the codes arising from the projective system determined by $\varepsilon_{k}^{g r}\left(\Delta_{k}\right)$ and determine some of their parameters. We also investigate special sets of points of $\Delta_{k}$ which are met by any line of $\Delta_{k}$ in at most 2 points proving that their image under the Grassmann embedding is a projective cap.
\end{abstract}

Keywords: Polar spaces, orthogonal Grassmannians, Dual polar spaces, embeddings, caps, error correcting codes.

1 Email: ilaria.cardinali@unisi.it

2 Email: giuzzi@ing.unibs.it 


\section{Introduction}

This note contains a quick preview of some new results on caps and linear error correcting codes related to the Grassmann embedding $\varepsilon_{k}^{g r}$ of orthogonal Grassmannians. These results will be presented in full in a forthcoming paper [4]. In Section 2 we provide some preliminaries on the topic; in particular, Subsection 2.1 recalls some properties of orthogonal Grassmannians, while codes arising from projective systems are discussed in Subsection 2.2. Our results are outlined in Section 3.

\section{Preliminaries}

\subsection{Orthogonal Grassmannians and their embeddings}

Let $V:=V(2 n+1, q)$ be a $(2 n+1)$-dimensional vector space over a finite field $\mathbb{F}_{q}$ endowed with a non-singular quadratic form $\eta$ of Witt index $n$. For $1 \leq k \leq n$ denote by $\mathcal{G}_{k}$ the $k$-Grassmannian of $\mathrm{PG}(V)$ and by $\Delta_{k}$ its $k-$ polar Grassmannian. Recall that the $k$-polar Grassmannian $\Delta_{k}$ is the proper subgeometry of $\mathcal{G}_{k}$ whose points are the $k$-subspaces of $V$ which are totally singular for $\eta$; the lines of $\Delta_{k}$ are

- for $k<n: \ell_{X, Y}:=\{Z \mid X \subset Z \subset Y$, $\operatorname{dim}(Z)=k\}$, with $\operatorname{dim} X=k-1$, $\operatorname{dim} Y=k+1$ and $Y$ totally singular;

- for $k=n$ : $\ell_{X}:=\left\{Z \mid X \subset Z \subset X^{\perp}, \operatorname{dim}(Z)=n, Z\right.$ totally singular $\}$, with $X$ a totally singular $(n-1)$-subspace of $V$ and $X^{\perp}$ its orthogonal with respect to $\eta$.

When $k=n$ the points of $\ell_{X}$ form a conic in the projective plane $\mathrm{PG}\left(X^{\perp} / X\right)$. Clearly, $\Delta_{1}$ is just the orthogonal polar space of rank $n$ associated to $\eta$; the geometry $\Delta_{n}$ can be regarded as the dual of $\Delta_{1}$ and is thus called orthogonal dual polar space of rank $n$.

Given a point-line geometry $\Gamma=(\mathcal{P}, \mathcal{L})$ we say that an injective map $e: \mathcal{P} \rightarrow \mathrm{PG}(V)$ is a projective embedding of $\Gamma$ if the following two conditions hold:

(1) $\langle e(\mathcal{P})\rangle=\mathrm{PG}(V)$;

(2) $e$ maps any line of $\Gamma$ onto a projective line.

Following [20], (see also [5]), when condition (2) is replaced by

(2') e maps any line of $\Gamma$ onto a non-singular conic of $\mathrm{PG}(V)$,

we say that $e$ is a Veronese embedding of $\Gamma$. 
Let now $W_{k}:=\bigwedge^{k} V$. The Grassmann embedding $e_{k}^{g r}: \mathcal{G}_{k} \rightarrow \operatorname{PG}\left(W_{k}\right)$ maps the arbitrary $k$-subspace $\left\langle v_{1}, v_{2}, \ldots, v_{k}\right\rangle$ of $V$ (that is a point of $\mathcal{G}_{k}$ ) to the point $\left\langle v_{1} \wedge v_{2} \wedge \cdots \wedge v_{k}\right\rangle$ of $\mathrm{PG}\left(W_{k}\right)$. Let $\varepsilon_{k}^{g r}:=\left.e_{k}^{g r}\right|_{\Delta_{k}}$ be the restriction of $e_{k}^{g r}$ to $\Delta_{k}$. For $k<n$, the mapping $\varepsilon_{k}^{g r}$ is a projective embedding of $\Delta_{k}$ in the subspace $\mathrm{PG}\left(W_{k}^{g r}\right):=\left\langle\varepsilon_{k}^{g r}\left(\Delta_{k}\right)\right\rangle$ of $\mathrm{PG}\left(W_{k}\right)$ spanned by $\varepsilon_{k}^{g r}\left(\Delta_{k}\right)$. We call $\varepsilon_{k}^{g r}$ the Grassmann embedding of $\Delta_{k}$.

If $k=n$, then $\varepsilon_{n}^{g r}$ is a Veronese embedding and maps the lines of $\Delta_{n}$ onto non-singular conics of $\mathrm{PG}\left(W_{n}\right)$. The dual polar space $\Delta_{n}$ affords also a projective embedding of dimension $2^{n}$, namely the spin embedding $\varepsilon_{n}^{s p i n}$.

Suppose $\nu_{2^{n}}$ to be the usual quadratic Veronese map $\nu_{2^{n}}: V\left(2^{n}, \mathbb{F}\right) \rightarrow$ $V\left(\left(\begin{array}{c}2^{n}+1 \\ 2\end{array}\right), \mathbb{F}\right)$. It is well known that $\nu_{2^{n}}$ defines a Veronese embedding of the point-line geometry $\operatorname{PG}\left(2^{n}-1, \mathbb{F}\right)$ in $\operatorname{PG}\left(\left(\begin{array}{c}2^{n}+1 \\ 2\end{array}\right)-1, \mathbb{F}\right)$, which will also be denoted by $\nu_{2^{n}}$. The composition $\varepsilon_{n}^{\mathrm{vs}}:=\nu_{2^{n}} \cdot \varepsilon_{n}^{\text {spin }}$ is a Veronese embedding of $\Delta_{n}$ in a subspace $\mathrm{PG}\left(W_{n}^{\text {vs }}\right)$ of $\mathrm{PG}\left(\left(\begin{array}{c}2^{n}+1 \\ 2\end{array}\right)-1, \mathbb{F}\right)$ : it is called the Veronese-spin embedding of $\Delta_{n}$. Properties of Grassmann and Veronese-spin embedding, fundamental in order to obtain our results, are extensively investigated in [5] and [6].

\subsection{Projective systems and Codes}

Error correcting codes are an essential component to any efficient communication system, as they can be used in order to guarantee arbitrarily low probability of mistake in the reception of messages without requiring noisefree operation; see [13]. An $[N, K, d]_{q}$ projective system $\Omega$ is a set of $N$ points in $\operatorname{PG}(K-1, q)$ such that for any hyperplane $\Sigma$ of $\mathrm{PG}(K-1, q)$, we have $|\Omega \backslash \Sigma| \geq d$. Existence of $[N, K, d]_{q}$ projective systems is equivalent to that of projective linear codes with the same parameters. Indeed, given a projective system $\Omega=\left\{P_{1}, \ldots, P_{N}\right\}$, fix a reference system $\mathfrak{B}$ in $\operatorname{PG}(K-1, q)$ and consider the matrix $G$ whose columns are the coordinates of the points of $\Omega$ with respect to $\mathfrak{B}$. Then, $G$ is the generator matrix of an $[N, K, d]$ code over $\mathbb{F}_{q}$, say $\mathcal{C}=\mathcal{C}(\Omega)$, uniquely defined up to code equivalence. Furthermore, as any word of $\mathcal{C}(\Omega)$ is of the form $c=m G$ for some row vector $m \in \mathbb{F}_{q}^{K}$, it is straightforward to see that the number of zeroes in $c$ is the same as the number of points $x$ of $\Omega$ lying on the hyperplane of equation $m \cdot x=0$ where $m \cdot x=\sum_{i=1}^{K} m_{i} x_{i}$ and $m=\left(m_{i}\right)_{1}^{K}, x=\left(x_{i}\right)_{1}^{K}$. In particular, the minimum distance of $\mathcal{C}$ turns out to be $d=\min \{|\Omega|-|\Omega \cap \Sigma|: \Sigma$ is a hyperplane of $\operatorname{PG}(\mathrm{k}-1, \mathrm{q})\}$. This provides a geometric interpretation of the meaning of minimum distance.

The link between incidence structures $\mathcal{S}=(\mathcal{P}, \mathcal{L})$ and codes is deep and it dates at least to [15]; we refer the interested reader to [1,3] and [19] for more 
details. Traditionally, two basic approaches have proved to be most fruitful: either to consider the incidence matrix of a structure as a generator matrix for a binary code, see for instance [12], or to consider an embedding of $\mathcal{S}$ in a projective space and study the code arising from the projective system thus determined or its dual; see e.g. [2,11,7] for codes related to the Segre embedding [16].

Codes based on projective Grassmannians have been first introduced in [17] as generalisations of Reed-Muller codes of the first order; see also [18]. We refer to $[14,9,10]$ for some recent developments.

\section{Main results}

We investigate linear codes associated with the projective system $\varepsilon_{k}^{g r}\left(\Delta_{k}\right)$ determined by the image of the Grassmann embedding $\varepsilon_{k}^{g r}$ of $\Delta_{k}$ obtaining the following parameters.

Theorem 3.1 Let $\mathcal{C}_{k, n}$ be the code determined by the projective system of $\varepsilon_{k}^{g r}\left(\Delta_{k}\right)$ for $1 \leq k<n$. Then, the parameters of $\mathcal{C}_{k, n}$ are

$$
\begin{gathered}
N=\frac{\left(q^{n-k+1}+1\right)\left(q^{n-k+2}+1\right) \ldots\left(q^{n}+1\right)\left(q^{n-k+1}-1\right)\left(q^{n-k+2}-1\right) \ldots\left(q^{n}-1\right)}{(q-1)\left(q^{2}-1\right) \ldots\left(q^{k}-1\right)}, \\
K=\left\{\begin{array}{c}
\left(\begin{array}{c}
2 n+1 \\
k
\end{array}\right) \quad \text { for } q \text { odd } \\
\left(\begin{array}{c}
2 n+1 \\
k
\end{array}\right)-\left(\begin{array}{c}
2 n+1 \\
k-2
\end{array}\right) \text { for } q \text { even }
\end{array}, \quad d \geq 2 q^{k(n-k)}-1 .\right.
\end{gathered}
$$

As for the codes arising from dual polar spaces of small rank, we have the following result where the minimum distance is precisely computed.

Theorem 3.2 (i) The code $\mathcal{C}_{2,2}$ arising from a dual polar space of rank 2 has parameters

$$
N=\left(q^{2}+1\right)(q+1), \quad K=\left\{\begin{array}{l}
10 \text { for } q \text { odd } \\
9 \text { for } q \text { even }
\end{array}, \quad d=q^{2}(q-1) .\right.
$$

(ii) The code $\mathcal{C}_{3,3}$ arising from a dual polar space of rank 3 has parameters

$$
\begin{array}{ll}
N=\left(q^{3}+1\right)\left(q^{2}+1\right)(q+1), & K=35, d=q^{2}(q-1)\left(q^{3}-1\right) \quad \text { for } q \text { odd } \\
& \text { and } \\
N=\left(q^{3}+1\right)\left(q^{2}+1\right)(q+1), & K=28, \quad d=q^{5}(q-1) \quad \text { for } q \text { even. }
\end{array}
$$


In order to define polar $m$-caps of $\Delta_{k}$, the general notion of $(m, v)$-set of a partial linear space has been introduced. It has been shown that, under the Grassmann embedding, the points of a polar $m$-cap (a set having the property that it is met by any line of $\Delta_{k}$ it in at most 2 points) are mapped onto the points of a projective cap; see also [8] for other projective caps contained in Grassmannians.

Theorem 3.3 Suppose $1 \leq k \leq n$. Then,

(i) for any polar $m$-cap $\mathcal{C}$ of $\Delta_{k}$, its image $\varepsilon_{k}^{g r}(\mathcal{C})$ is a projective cap of $\mathrm{PG}\left(W_{k}\right)$;

(ii) the set $\varepsilon_{n}^{g r}\left(\Delta_{n}\right)$ is a projective cap.

We were also able to explicitly construct polar caps of $\Delta_{k}$ for $k \leq n$ and build a related design, as shown in the following.

Theorem 3.4 For any $r \leq\lfloor k / 2\rfloor$, the polar Grassmannian $\Delta_{k}$ contains a polar $2^{r}$-cap. This cap is explicitly determined and it is shown that it can be suitably represented by means of a Hadamard matrix in Sylvester form.

\section{References}

[1] Assmus, E.F., and J.D. Key., "Designs and their codes", Cambridge University Press, Cambridge (1992).

[2] Betten, A., Twisted Tensor Product Codes, Des. Codes Cryptogr. 47 (2008), no. 1-3, 191-219.

[3] Cameron, P.J., and J.H. van Lint, "Designs, Graph, Codes and their Links", Cambridge University Press (1991).

[4] Cardinali, I., and L. Giuzzi, Caps and codes from orthogonal Grassmannians, submitted.

[5] Cardinali, I., and A. Pasini, Veronesean embeddings of dual polar spaces of orthogonal type, submitted.

[6] Cardinali I., and A. Pasini, Grassmann and Weyl Embeddings of Orthogonal Grassmannians, submitted.

[7] Couvreur, A., and I. Duursma, Evaluation codes from smooth Quadric Surfaces and Twisted Segre Varieties, arXiv 1101.4603v1.

[8] Ebert, G., K. Metsch, and T. Szöni, Caps embedded in Grassmannians, Geom. Dedicata 70 (1998), 181-196. 
[9] Ghorpade, S.R., and G. Lachaud, Hyperplane sections of Grassmannians and the number of MDS linear codes, Finite Fields Appl. 7 (2001), 468-506.

[10] Ghorpade, S.R., A.R. Patir, and H.K. Pillai, Decomposable subspaces, linear sections of Grassmann varieties, and Higher weights of Grassmann codes, Finite Fields Appl. 15 (2009), 54-68.

[11] Giuzzi, L., and V. Pepe, Families of twisted tensor product codes, Des. Codes Cryptogr., to appear.

[12] Giuzzi, L., and A. Sonnino, LDPC codes from Singer Cycles, Discrete Appl. Math. 157 (8) (2009), 1723-1728.

[13] MacWilliams, F.J., and N.J.A. Sloane, "The theory of error correcting codes", North-Holland Publishing Co., Amsterdam-New York-Oxford (1977).

[14] Nogin, D.Yu., Codes associated to Grassmannians, in "Arithmetic, geometry and coding theory (Luminy, 1993)", de Gruyter (1996), 145-154.

[15] Paige, L.J., A note on the Mathieu groups, Canad. J. Math. 9 (1957), 15-18.

[16] Pepe, V., On the algebraic variety $\mathcal{V}_{r, t}$, Finite Fields Appl. 17 (2011), 343-349.

[17] Ryan, C.T., An application of Grassmannian varieties to coding theory, Congr. Numer. 57 (1987), 257-271.

[18] Ryan, C.T., Projective codes based on Grassmann varieties, Congr. Numer. 57 (1987), 273-279.

[19] Tang, H., J. Xu, S. Lin, and K.A.S. Abded-Ghaffar, Codes on Finite Geometries, IEEE Trans. Inform. Theory 51, No. 2 (2005), 572-596.

[20] Thas, J.A., and H. Van Maldeghem, Generalized Veronesean embeddings of projective spaces, Combinatorica 31 (5) (2011), 615-629. 